\title{
Magnetic Compton Scattering on Sr-Doped $\mathrm{LaCoO}_{3}$
}

\author{
Yoshihiko Kobayashi ${ }^{1, *} \mathbb{*}$, Naruki Tsuji ${ }^{2}$ and Yoshiharu Sakurai ${ }^{2} \mathbb{D}$ \\ 1 Department of Physics, Tokyo Medical University, Shinjuku, Tokyo 160-8402, Japan \\ 2 Japan Synchrotron Radiation Research Institute (JASRI), SPring-8, 1-1-1 Kouto, Sayo 679-5198, Japan; \\ ntsuji@spring8.or.jp (N.T.); sakurai@spring8.or.jp (Y.S.) \\ * Correspondence: koba@tokyo-med.ac.jp; Tel.: +81-3-3351-6141 (ext. 252)
}

\section{check for}

updates

Citation: Kobayashi, Y.; Tsuji, N.; Sakurai, Y. Magnetic Compton Scattering on Sr-Doped $\mathrm{LaCoO}_{3}$. Crystals 2021, 11, 577. https:// doi.org/10.3390/cryst11060577

Academic Editor: Borislav Angelov

Received: 23 April 2021

Accepted: 19 May 2021

Published: 21 May 2021

Publisher's Note: MDPI stays neutral with regard to jurisdictional claims in published maps and institutional affiliations.

Copyright: (c) 2021 by the authors. Licensee MDPI, Basel, Switzerland. This article is an open access article distributed under the terms and conditions of the Creative Commons Attribution (CC BY) license (https:/ / creativecommons.org/licenses/by/ $4.0 /)$.

\begin{abstract}
We carried out magnetic Compton scattering experiments on the Sr-doped perovskite cobaltite $\mathrm{La}_{1-x} \mathrm{Sr}_{x} \mathrm{CoO}_{3}$ for the paramagnetic insulator $(x=0.1)$ and the ferromagnetic metallic $(x=0.3)$ phases in order to investigate the electron-orbital state relevant to its unusual magnetic and electronic transport properties. The ratio of the orbital moments to the spin moments $\left(M_{\text {orbital }} / M_{\text {spin }}\right)$ is less than $1 / 10$ at low temperatures for $x=0.3$, whereas $M_{\text {orbital }} / M_{\text {spin }}$ is about 0.4 almost independent of temperature for $x=0.1$. The shape of $J_{\text {mag }}\left(p_{z}\right)$ shows no apparent difference between the paramagnetic insulator and the ferromagnetic metallic phases.
\end{abstract}

Keywords: Sr-doped $\mathrm{LaCoO}_{3}$; magnetic ground state; spin-state; orbital moment; magnetic Compton scattering

PACS: 75.20.Hr; 75.30.Mb; 71.23.-k

\section{Introduction}

Perovskite cobaltites have been attracting the widespread interest of researchers because of their exotic physical and chemical properties, which are attributed to the multiple degrees of freedom on the spin, orbital, and lattice arising from the $3 d^{6}$ electronic configuration of $\mathrm{Co}^{3+}[1-4]$. One of the examples is the unusual spin-crossover phenomena of $\mathrm{LaCoO}_{3}$, in which broad magnetic anomalies take place at around 100 and $500 \mathrm{~K}$ [5]. The magnetic anomaly at around $500 \mathrm{~K}$ is accompanied by an insulator-to-metal transition (IMT). These phenomena are considered to be the temperature-induced spin-crossover from the nonmagnetic low-spin (LS; $\left.\left(t_{2 g} \uparrow\right)^{3}\left(t_{2 g} \downarrow\right)^{3}, S=0\right)$ ground state to magnetic excited states ( $\uparrow$ and $\downarrow$ denote up and down spins, respectively) [6]. Despite the decades of research history and the extensive efforts of researchers, there is still controversy regarding whether the magnetic excited state induced around $100 \mathrm{~K}$ is intermediate spin (IS; $\left(t_{2 g} \uparrow\right)^{3}\left(e_{g} \uparrow\right)^{1}\left(t_{2 g} \downarrow\right)^{2}$, $S=1$ ) or high spin (HS; $\left.\left(t_{2 g} \uparrow\right)^{3}\left(e_{g} \uparrow\right)^{2}\left(t_{2 g} \downarrow\right)^{1}, S=2\right)$. Neither spin-state model can comprehensively explain all the important experimental findings [6-11].

It has been reported that the introduction of $\mathrm{Co}^{4+}$ and the positive holes by the substitution of $\mathrm{Sr}^{2+}$ into $\mathrm{La}^{3+}$ site in $\mathrm{LaCoO}_{3}$ brings about the disappearance of LS ground state and the appearance of the magnetic ground state [1,12-15]. This fact means that not only the doped-Co ${ }^{4+}$ but also the rest of $\mathrm{Co}^{3+}$ ions are converted into the magnetic state in $\mathrm{La}_{1-x} \mathrm{Sr}_{x} \mathrm{CoO}_{3}$. The previous experiments show that the paramagnetic insulator phase is realized for $0<x \leq \sim 0.2$, and the cluster-glass-like ferromagnetic metallic phase appears for $\sim 0.2<x \leq \sim 0.5$ [12-15]. The fact that the hole-doping drastically changes the magnetic and transport properties of perovskite cobaltite implies that the subtle change of the environment of Co ions varies the orbital-spin state of Co-3d in perovskite cobaltites.

The magnetic ground state of $\mathrm{La}_{1-x} \mathrm{Sr}_{x} \mathrm{CoO}_{3}$ is considered to be IS state, although the experimental basis is insufficient. If the IS ground state is realized in $\mathrm{Sr}$-doped $\mathrm{LaCoO}_{3}$, significant magnitudes of orbital moments are expected to be observed since the IS state $\left(e_{g}^{1} t_{2 g}^{5}\right.$ for $\left.\mathrm{Co}^{3+}\right)$ has a degree of freedom on both $e_{g}$ and $t_{2 g}$ orbitals. 
As described above, detailed experimental studies on the electronic structure of Co-3d are desired to understand the unusual magnetic and electronic transport properties in perovskite cobaltite. In this paper, we report magnetic Compton scattering experiments on $\mathrm{La}_{1-x} \mathrm{Sr}_{x} \mathrm{CoO}_{3}$ in order to investigate the orbital-spin state of Co-3d responsible for the magnetic and electronic transport properties of Sr-doped $\mathrm{LaCoO}_{3}$ for $x=0.1$ and 0.3. The X-ray Compton scattering experiments provide imaging of the electron distribution in the momentum space through Compton profiles $J\left(p_{z}\right)$, which exhibit projection of the electron momentum density $\rho(p)\left[p \equiv\left(p_{x}, p_{y}, p_{z}\right)\right]$ onto the $z$-axis along the scattering vector [16], as

$$
J\left(p_{z}\right)=\iint \rho(\boldsymbol{p}) d p_{x} d p_{y}
$$

The magnetic Compton profiles $J_{m a g}\left(p_{z}\right)$ show the projection of the spin-dependent electron momentum density onto the $z$ axis as

$$
J_{m a g}\left(p_{z}\right)=\iint\left(\rho_{\uparrow}(\boldsymbol{p})-\rho_{\downarrow}(\boldsymbol{p})\right) d p_{x} d p_{y}
$$

where $\rho_{\uparrow}(\boldsymbol{p})$ and $\rho_{\downarrow}(\boldsymbol{p})$ denote the electron momentum density with the majority and the minority spins, respectively [16-18]. The magnetic Compton scattering enables evaluating the spin magnetic moments of the materials from the integral (area intensity) of $J_{m a g}\left(p_{z}\right)$, as can be easily understood from Equation (2). The orbital moments can be obtained by extracting the spin moments from the total magnetic moments measured by the magnetization measurements. If a non-negligible magnitude of the orbital moments are observed in $\mathrm{La}_{1-x} \mathrm{Sr}_{x} \mathrm{CoO}_{3}$, it strongly supports the IS state as the magnetic ground state. Furthermore, the difference of the electronic-orbital state of Co-3d due to the itineracy and/or the magnetic ordering of Co-3 $d$ electrons might be observed by the magnetic Compton scattering experiments. $\mathrm{La}_{1-x} \mathrm{Sr}_{x} \mathrm{CoO}_{3}$ changes its magnetic and transport properties with changing the amount of doped holes [12-15]. If the electronic-orbital state of Co-3d is significantly different between the paramagnetic insulator $(x=0.1)$ and the ferromagnetic metallic $(x=0.3)$ phases of $\mathrm{La}_{1-x} \mathrm{Sr}_{x} \mathrm{CoO}_{3}$, it is possible to reflect the difference of the Co- $3 d$ electron-orbital state in the shape of $J_{m a g}\left(p_{z}\right)$ [16-18].

\section{Materials and Methods (Experimental Procedure)}

The polycrystalline samples of $\mathrm{La}_{1-x} \mathrm{Sr}_{x} \mathrm{CoO}_{3}(x=0.1$ and 0.3$)$ were prepared by solid-phase reaction of $\mathrm{La}_{2} \mathrm{O}_{3}(99.99 \%), \mathrm{SrCO}_{3}(99.9 \%)$, and $\mathrm{Co}_{3} \mathrm{O}_{4}(99.9 \%)$. After a mixture of the starting materials with an appropriate proportion was prefired at $900{ }^{\circ} \mathrm{C}$ for $24 \mathrm{~h}$ in oxygen gas flow, the mixture was ground, pressed into pellets, fired again at $1300{ }^{\circ} \mathrm{C}$ in oxygen gas flow for $48 \mathrm{~h}$, and then subjected to furnace-cooling. X-ray powder diffraction measurements were performed with $\mathrm{Cu}-\mathrm{K} \alpha$ radiation at room temperature. Powder $\mathrm{X}$-ray profiles clarified the crystals to be of single phase. The measurements of temperature dependence of magnetization, by using a Quantum Design SQUID magnetometer between 2 to $400 \mathrm{~K}$ under the magnetic field $2.5 \mathrm{~T}$, confirmed that the sample with $x=0.1$ is paramagnetic, and that with $x=0.3$, it is ferromagnetic with a ferromagnetic Curie temperature $\left(T_{\mathcal{C}}\right)$ of about $220 \mathrm{~K}$. The field dependence of the magnetization below $T_{\mathcal{C}}$ exhibits that the magnitude of the saturation magnetization is about $1.1 \mu_{\mathrm{B}} /$ Co for $x=0.3$.

The magnetic Compton scattering experiments were conducted using the beam line BL08W at SPring-8, Japan. Elliptically polarized X-rays were monochromatized to be $182.6 \mathrm{keV}$ and focused to a spot of about $1 \times 0.8 \mathrm{~mm}^{2}$ on the samples. The scattered X-rays with a scattering angle of $174^{\circ}$ were energy analyzed by a 10-segmented Ge solid state detector. During the measurements, to reverse the direction of magnetization in the sample, an external magnetic field of $\pm 2.5 \mathrm{~T}$ was alternatively applied along the $z$-axis. The $J_{\text {mag }}\left(p_{z}\right)$ was extracted as the difference in Compton scattering intensities in reversing the direction of the external field to a fixed photon polarization as

$$
J_{\text {mag }}\left(p_{z}\right)=J^{+}\left(p_{z}\right)-J^{-}\left(p_{z}\right),
$$


where $J^{+}\left(p_{z}\right)$ and $J^{-}\left(p_{z}\right)$ denote the Compton profiles for the magnetic field parallel to the $z$-axis and antiparallel to the $z$-axis, respectively [16]. The spin electron number $n_{\text {mag }}$ is obtained from

$$
\begin{gathered}
n_{\text {mag }}=\frac{R}{R_{\mathrm{Fe}}} \frac{n_{\text {total }}}{n_{\text {total }}^{\mathrm{Fe}}} n_{\text {mag }}^{\mathrm{Fe}} \\
R=\frac{A_{\text {spin }}^{+} n_{\text {mag }}}{A_{\text {nor }} n_{\text {total }}}, \\
R_{\mathrm{Fe}}=\frac{A_{\text {spin }}^{+} n_{\text {mag }}^{\mathrm{Fe}}}{A_{\text {nor }} n_{\text {total }}^{\mathrm{Fe}}},
\end{gathered}
$$

where $R$ denotes the magnetic effect with the Compton scattering cross-section $A$ and the electron number $n$ corresponding to the quantity represented by the subscripts and superscripts [16]. In order to evaluate the spin moments, the area intensity of measured $J_{m a g}\left(p_{z}\right)$ was normalized using the eqationss described above and the reference Fe sample with the spin moment $2.08 \mu_{\mathrm{B}} / \mathrm{Fe}$ at room temperature and at $2.5 \mathrm{~T}$ [19].

\section{Results}

Figure 1 shows the $J_{m a g}\left(p_{z}\right)$ of $\mathrm{La}_{0.7} \mathrm{Sr}_{0.3} \mathrm{CoO}_{3}$ measured at $T=10 \mathrm{~K}$ and $B=2.5 \mathrm{~T}$. The calculated Compton profiles $\left(J\left(p_{z}\right)\right)$ of Co-3d is also shown for comparison, in which the calculated $J\left(p_{z}\right)$ was evaluated by the sum of the $J\left(p_{z}\right)$ of the wave functions $e_{g}\left(x^{2}-y^{2}\right.$ and $\left.3 z^{2}-r^{2}\right)$ and $t_{2 g}(x y, y z$ and $z x)$ calculated using Equation (1), assuming that the contents of $\mathrm{Co}^{3+}$ and $\mathrm{Co}^{4+}$ are 0.7 and 0.3 , respectively, and the LS Co ${ }^{4+}\left(e_{g}^{0} t_{2 g}^{5}\right)$, IS $\left(e_{g}^{1} t_{2 g}^{5}\right.$ for $\mathrm{Co}^{3+}$ and $e_{g}^{1} t_{2 g}^{4}$ for $\left.\mathrm{Co}^{4+}\right)$ and HS $\left(e_{g}^{2} t_{2 g}^{4}\right.$ for $\mathrm{Co}^{3+}$ and $e_{g}^{2} t_{2 g}^{3}$ for $\left.\mathrm{Co}^{4+}\right)$ exist without weight. The calculated $J\left(p_{z}\right)$ satisfactorily reproduces the measured $J_{m a g}\left(p_{z}\right)$ for $p_{z}>\sim 1.3$, suggesting that the Co- $3 d$ orbitals are responsible for the spin magnetic moments. The deviation for $p_{z}<\sim 1.3$ might be due to the polarization of inner-shell electrons and/or the hybridization between Co-3d and O-2 $p$ orbitals $[20,21]$. The magnitude of the $J_{\text {mag }}\left(p_{z}\right)$ decreases with increasing temperature, maintaining its shape. These characteristics of the $J_{\text {mag }}\left(p_{z}\right)$ are basically the same as those of $\mathrm{La}_{0.9} \mathrm{Sr}_{0.1} \mathrm{CoO}_{3}$.

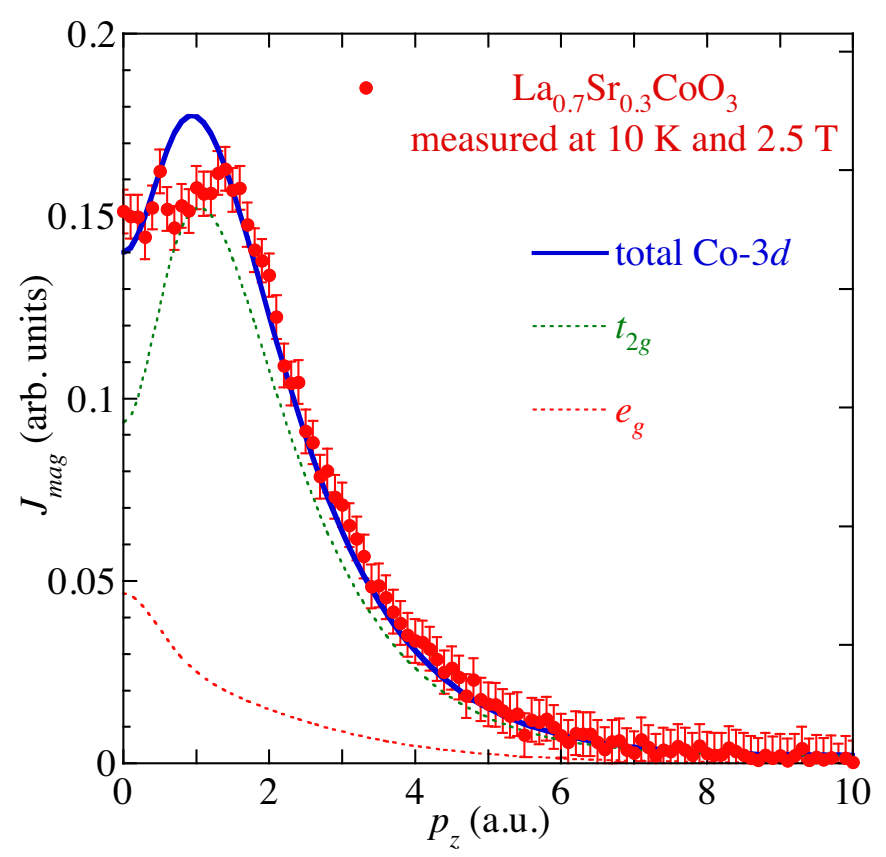

Figure 1. The magnetic Compton scattering profile $\left(J_{m a g}\left(p_{z}\right)\right)$ of $\mathrm{La}_{0.7} \mathrm{Sr}_{0.3} \mathrm{CoO}_{3}$ measured at $T=10 \mathrm{~K}$ and $B=2.5 \mathrm{~T}$, along with the calculated Compton profiles $\left(J\left(p_{z}\right)\right)$ of Co-3d. 
The magnitude of the spin moments $\left(M_{\text {spin }}\right)$ were evaluated by the area intensity of $J_{\text {mag }}\left(p_{z}\right)$. The orbital moments $\left(M_{\text {orbital }}\right)$ were obtained by extracting the $M_{\text {spin }}$ from the total magnetic moment $\left(M_{\text {total }}\right)$ measured by the magnetization measurements. The temperature dependence of $M_{\text {total }}, M_{\text {spin }}$, and $M_{\text {orbital }}\left(=M_{\text {total }}-M_{\text {spin }}\right)$ is shown in Figure 2a,c for $\mathrm{La}_{1-x} \mathrm{Sr}_{x} \mathrm{CoO}_{3}$ with $x=0.3$ and 0.1 , respectively. The temperature dependence of $M_{\text {spin }}$ mimics that of $M_{\text {total }}$, and the magnitude of $M_{\text {spin }}$ accounts for the majority of the magnitude of $M_{\text {total }}$ for all the temperature region for both $x=0.3$ and 0.1, exhibiting that the spin components are dominant in the magnetization in $\mathrm{La}_{1-x} \mathrm{Sr}_{x} \mathrm{CoO}_{3}$. This finding is reasonably understood as the magnetism of $3 d$-electrons under the cubic-symmetrical crystalline-electric-field, in which the orbital angular momentum almost vanishes, although the non-negligible magnitude of the orbital components are observed for all the temperature region for both $x=0.3$ and 0.1 .

In order to evaluate the contribution of the orbital components to the magnetization, the ratio of $M_{\text {orbital }}$ to $M_{\text {spin }}\left(M_{\text {orbital }} / M_{\text {spin }}\right)$ is shown in Figure $2 \mathrm{~b}, \mathrm{~d}$ for $x=0.3$ and 0.1 , respectively. For $x=0.3, M_{\text {orbital }} / M_{\text {spin }}$ is significantly small $\left(M_{\text {orbital }} / M_{\text {spin }}=\sim 1 / 10\right)$ at lower temperatures below $T_{c}$, and increases with increasing temperature above $\sim 150 \mathrm{~K}$, reflecting the abrupt decrease of $M_{\text {spin }}$ with increasing temperature at around the $T_{c}$. For $x=0.1, M_{\text {orbital }} / M_{\text {spin }}$ is about 0.4 in the entire temperature region, and no pronounced temperature dependence was observed, although the experimental errors are large due to the smallness of both $M_{\text {spin }}$ and $M_{\text {orbital }}$.
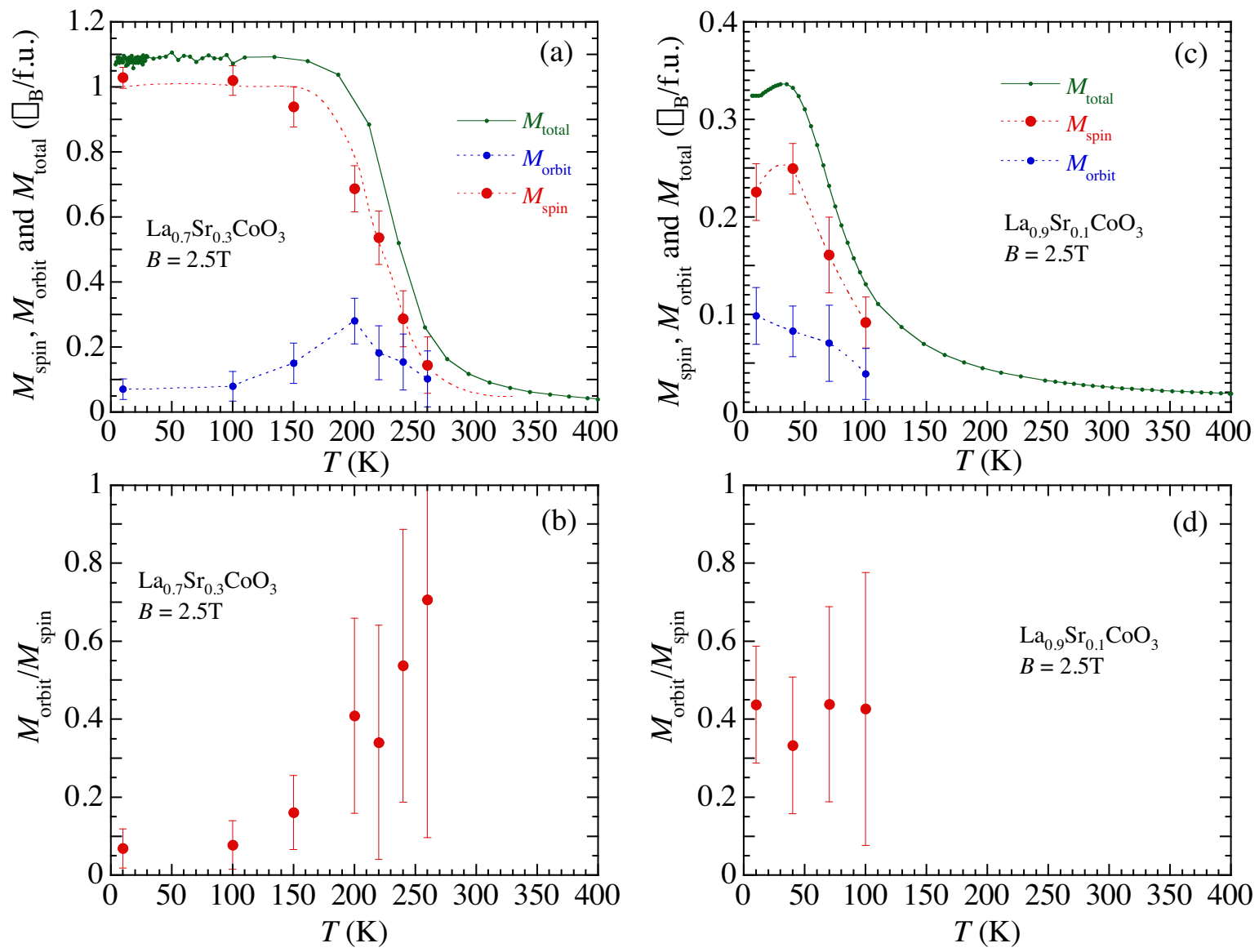

Figure 2. (a) Temperature dependence of the total magnetization $\left(M_{\text {total }}\right)$ measured by the magnetization measurements, spin moments $\left(M_{\text {spin }}\right)$ obtained from $J_{m a g}\left(p_{z}\right)$, and orbital moments $\left(M_{\text {orbital }}\right)$ extracted by $M_{\text {total }}-M_{\text {spin }}$, and (b) temperature dependence of the ratio of $M_{\text {orbital }}$ to $M_{\text {spin }}\left(M_{\text {orbital }} / M_{\text {spin }}\right)$ for $\mathrm{La}_{0.7} \mathrm{Sr}_{0.3} \mathrm{CoO}_{3}$. (c) Temperature dependence of $M_{\text {total }}$, $M_{\text {spin, }}$ and $M_{\text {orbital }}$ and (d) $M_{\text {orbital }} / M_{\text {spin }}$ for $\mathrm{La}_{0.9} \mathrm{Sr}_{0.1} \mathrm{CoO}_{3}$. 
In order to examine whether the temperature change of the electron-orbital state appears in $J_{m a g}\left(p_{z}\right)$, we compared the shape of $J_{m a g}\left(p_{z}\right)$ measured at different temperatures for each sample, as shown in Figure 3. Each $J_{\text {mag }}\left(p_{z}\right)$ was normalized to have the same area intensity so that the shapes of $J_{\text {mag }}\left(p_{z}\right)$ can be compared. The shape of $J_{\text {mag }}\left(p_{z}\right)$ is almost the same for all the temperatures measured for both samples, although the scatter of data points is fairly large at higher temperatures. This finding suggests that $\mathrm{Sr}$-doped $\mathrm{LaCoO}_{3}$ has no distinct change in the electron-orbital states due to temperature change. From the comparison of the shape of $J_{m a g}\left(p_{z}\right)$ between the Figure $3 \mathrm{a}, \mathrm{b}$, the electron-orbital states responsible for the spin moments are basically the same between the paramagnetic insulator phase $(x=0.1)$ and the ferromagnetic metallic phase $(x=0.3)$ within the experimental accuracy.
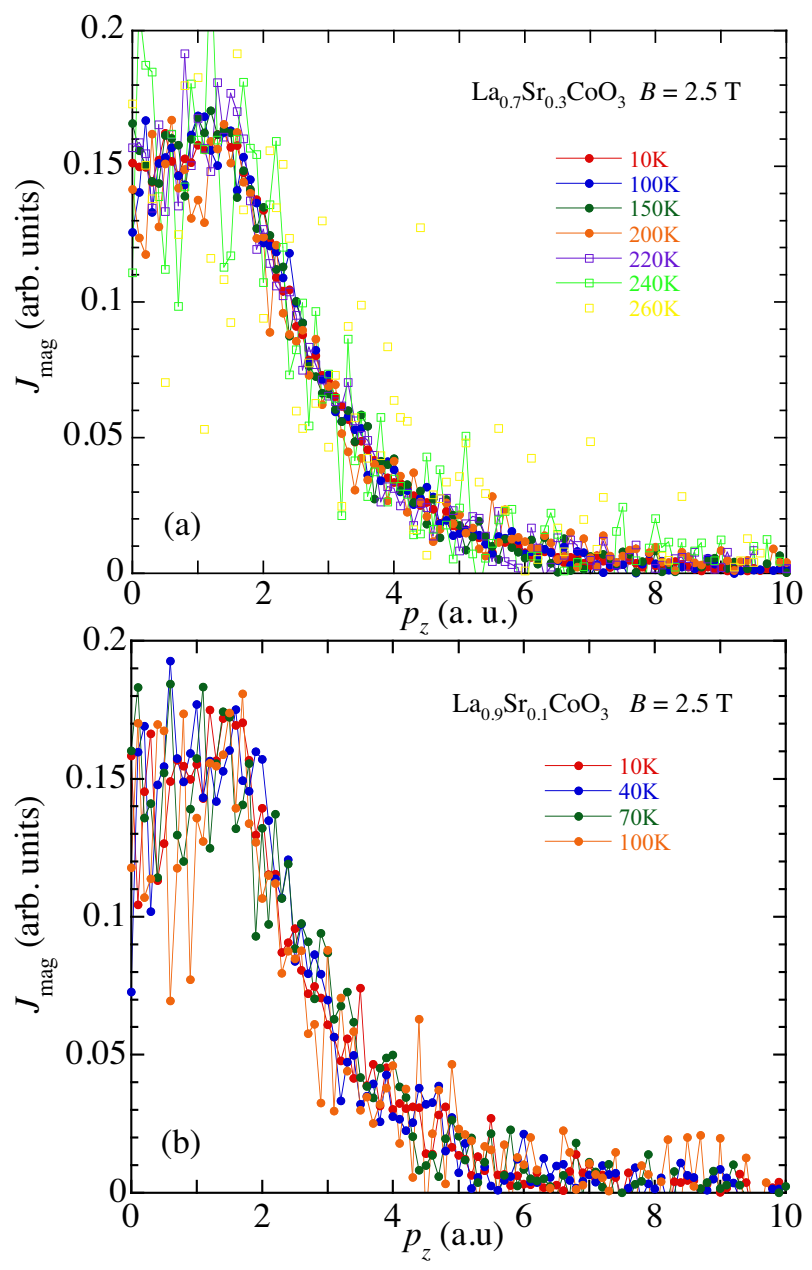

Figure 3. The $J_{m a g}\left(p_{z}\right)$ of (a) $\mathrm{La}_{0.7} \mathrm{Sr}_{0.3} \mathrm{CoO}_{3}$ measured at temperatures from 10 to $260 \mathrm{~K}$, and (b) $\mathrm{La}_{0.9} \mathrm{Sr}_{0.1} \mathrm{CoO}_{3}$ measured at temperatures from 10 to $100 \mathrm{~K}$. The $J_{m a g}\left(p_{z}\right)$ was normalized to have the same area intensity. The error bars were omitted for better clarity.

\section{Discussion}

The ratio $M_{\text {orbital }} / M_{\text {spin }}$ is $\sim 1 / 10$ at lower temperatures below $T_{c}$ for $x=0.3$, whereas $M_{\text {orbital }} / M_{\text {spin }}$ is $\sim 0.4$ almost independent of temperature for $x=0.1$ as shown in Figure $2 \mathrm{~b}, \mathrm{~d}$, respectively. The difference in $M_{\text {orbital }} / M_{\text {spin }}$ implies that the itineracy and/or magnetic ordering is pertinent to the magnitude of the remaining orbital angular momentum. The presence of orbital moments of significant magnitude for $x=0.1$ indicate that the comparatively large orbital component remains for the paramagnetic insulator phase. Both the $e_{g}$ 
and $t_{2 g}$ orbitals have a degree of freedom for both $\mathrm{IS}^{3+} \mathrm{Co}^{3+}$ and $\mathrm{Co}^{4+}$. The calculated ratio $M_{\text {orbital }} / M_{\text {spin }}$ is $\sim 0.33$ for IS $\mathrm{Co}^{3+}, \sim 0.5$ for IS $\mathrm{Co}^{4+}, \sim 0.2$ for $\mathrm{HS} \mathrm{Co}^{3+}$ and $\sim 0.02$ for HS $\mathrm{Co}^{4+}$ [22]. Thus, the experimental values $\left(M_{\text {orbital }} / M_{\text {spin }} \sim 0.4\right)$ can be roughly explained if we consider a mixture of IS $\mathrm{Co}^{3+}$ and $\mathrm{IS} \mathrm{Co}^{4+}$. The effective magnetic moment has been reported as $p_{\text {eff }}=2.9 \mu_{\mathrm{B}} / \mathrm{Co}$ in $\mathrm{La}_{0.9} \mathrm{Sr}_{0.1} \mathrm{CoO}_{3}$, which is close to the values expected for IS $\mathrm{Co}^{3+}$ [14]. Thus, the orbital moments in the present magnetic Compton scattering experiments along with the magnitude of $p_{e f f}$ suggest the IS ground state in the paramagnetic insulator phase of Sr-doped $\mathrm{LaCoO}_{3}$.

On the other hand, $M_{\text {orbital }} / M_{\text {spin }}$ for $x=0.3$ is considerably small, suggesting that the spin component is dominant in the magnetization and the orbital component is significantly small for the ferromagnetic metallic phase. The magnitude of the saturation magnetization about $1.1 \mu_{\mathrm{B}} / \mathrm{Co}$ is much smaller than that expected from both IS and HS states of $\mathrm{Co}^{3+}$ ions, even taking into account the coexistence of $\mathrm{Co}^{4+}$. These characteristics in the magnetism seem to be qualitatively different from those for the paramagnetic insulator phase $(x=0.1)$. Okamoto et al. investigated the magnetic moment of $\mathrm{Co}$ in $\mathrm{La}_{1-x} \mathrm{Sr}_{x} \mathrm{CoO}_{3}$ using the magnetic circular X-ray dichroism (MCXD) and magnetization measurements, and they also reported the smallness of the magnetization in the ferromagnetic metallic phase [22]. In their report, they attributed the smallness of the magnetic moment to the itineracy of the Co-3d electrons. However, their MCXD measurements exhibit that $M_{\text {orbital }} / M_{\text {spin }}$ is almost independent of the $\mathrm{Sr}$ content, suggesting that the contribution of the orbital moments to the magnetization is almost the same between the paramagnetic insulator and the ferromagnetic metallic phases, in contrast to the results of the magnetic Compton scattering experiments [22]. There might be a limitation in applying the spin sum rule, which was used to extract the spin moment of this substance in the MCXD measurement.

As shown in Figure $3 \mathrm{a}, \mathrm{b}$, the difference in the shape of $J_{\text {mag }}\left(p_{z}\right)$ with changing temperature and/or Sr content was not observed. This finding is noteworthy because it means that there is no remarkable difference in the electron-orbital state responsible for the spin magnetic moments despite the significant difference in magnitude of the orbital components between the paramagnetic insulator and the ferromagnetic metallic phases. The difference in the electron-orbital states between the paramagnetic localized electrons and the ferromagnetic itinerant electrons may be reflected only as a subtle difference in the shape of $J_{\text {mag }}\left(p_{z}\right)$. High-resolution magnetic Compton experiments are indispensable for evaluating the difference in electron-orbital states of Co-3d in $\mathrm{La}_{1-x} \mathrm{Sr}_{x} \mathrm{CoO}_{3}$ by using the shape of $J_{\text {mag }}\left(p_{z}\right)$.

\section{Conclusions}

We carried out magnetic Compton scattering experiments on the perovskite cobaltite $\mathrm{La}_{1-x} \mathrm{Sr}_{x} \mathrm{CoO}_{3}$ of the paramagnetic insulator $(x=0.1)$ and the ferromagnetic metallic $(x=0.3)$ phases in order to investigate the electron-orbital state responsible for the unusual magnetic and electronic transport properties. The measured $J_{m a g}\left(p_{z}\right)$ is almost reproduced by that of the ionic Co-3d orbitals under the cubic-symmetrical crystalline-electric-field ( $e_{g}$ and $t_{2 g}$ ) for both $x=0.1$ and 0.3. The non-negligible magnitude of the orbital moments were observed for both $x=0.1$ and 0.3 . The orbital component is significantly small for $x=0.3\left(M_{\text {orbital }} / M_{\text {spin }} \sim 1 / 10\right)$, whereas a comparatively large orbital moment $\left(M_{\text {orbital }} / M_{\text {spin }} \sim 0.4\right)$ independent of temperature is observed. This finding implies the difference between the IS state of local Co-3d for the paramagnetic insulator and the itinerant electrons for the ferromagnetic metallic phases, although no remarkable difference between $x=0.1$ and 0.3 was observed in the shape of $J_{m a g}\left(p_{z}\right)$ within the experimental accuracy of the present magnetic Compton scattering measurements.

Author Contributions: All authors made critical comments on the manuscript. Y.K. synthesized the samples and performed the magnetization measurements. Y.K., Y.S. and N.T. carried out the magnetic Compton scattering experiments and the analysis of the data. All authors have read and agreed to the published version of the manuscript. 
Funding: This research received no external funding.

Institutional Review Board Statement: Not applicable.

Informed Consent Statement: Not applicable.

Acknowledgments: The authors thank M. Itou for his experimental assistance. The Compton scattering experiments were performed with the approval of the Japan Synchrotron Radiation Research Institute (JASRI), SPring-8 (Proposals No. 2011B1298, No. 2012A1714, No. 2013A1023, No. 2014B1201, No. 2015A1211, No. 2016B1067, No. 2017A1175, No. 2018B1193, and No. 2021A1451).

Conflicts of Interest: The authors declare no conflict of interest.

\section{References}

1. Goodenough, J.B.; Longo, J.M. Landort-Börunstein; Springer: Berlin/Heidelberg, Germany, 1970; Volume 4a.

2. Imada, M.; Fujimori, A.; Tokura, Y. Metal-insulator transitions. Rev. Mod. Phys. 1998, 70, 1039. [CrossRef]

3. Kundu, A.K. (Ed.) Magnetic Perovskites—Synthesis, Structure and Physical Properties; Springer: Berlin/Heidelberg, Germany, 2016.

4. Bussmann-Holder, A.; Keller, H. (Eds.) High $T_{c}$ Superconductors and Related Transition Metal Oxides; Springer: Berlin/Heidelberg, Germany, 2007.

5. Goodenough, J.B. An interpretation of the magnetic properties of the perovskite-type mixed crystals $L a_{1-x} S r x C o O_{3-\lambda}$. J. Phys. Chem. Solids 1958, 6, 287. [CrossRef]

6. Asai, K.; Yoneda, A.; Yokokura, O.; Tranquada, J.M.; Shirane, G.; Kohn, K. Two Spin-State Transitions in LaCoO 3 . J. Phys. Soc. Jpn. 1998, 67, 290. [CrossRef]

7. Saitoh, T.; Mizokawa, T.; Fujimori, A.; Abbate, M.; Takeda, Y.; Takano, M. Electronic structure and temperature-induced paramagnetism in $\mathrm{LaCoO}_{3}$. Phys. Rev. B 1997, 55, 4257. [CrossRef]

8. Noguchi, S.; Kawamata, S.; Okuda, K.; Nojiri, H.; Motokawa, M. Evidence for the excited triplet of $\mathrm{Co}^{3+}$ in $\mathrm{LaCoO}_{3}$. Phys. Rev. B 2002, 66, 094404. [CrossRef]

9. Kyômen, T.; Asaka, Y.; Itoh, M. Negative cooperative effect on the spin-state excitation in $\mathrm{LaCoO}_{3}$. Phys. Rev. B 2003, 67, 144424 . [CrossRef]

10. Kyômen, T.; Asaka, Y.; Itoh, M. Thermodynamical analysis of spin-state transitions in $\mathrm{LaCoO}_{3}$ : Negative energy of mixing to assist thermal excitation to the high-spin excited state. Phys. Rev. B 2005, 71, 024418. [CrossRef]

11. Sato, K.; Matsuo, A.; Kindo, K.; Kobayashi, Y.; Asai, K. Field Induced Spin-State Transition in LaCoO 3 . J. Phys. Soc. Jpn. 2009, 78, 093702. [CrossRef]

12. Gerthsen, V.P.; Härdil, K.H. Halbleitereigenschaften des Lanthankobaltit. Z. Naturforsch. 1962, 17a, 514. [CrossRef]

13. Itoh, M.; Natori, I.; Kubota, S.; Motoya, K. Spin-Glass Behavior and Magnetic Phase Diagram of $\operatorname{La}_{1-x} \mathrm{Sr}_{x} \mathrm{CoO}_{3}(0 \leq x \leq 0.5)$ Studied by Magnetization Measurements. J. Phys. Soc. Jpn. 1994, 63, 1486. [CrossRef]

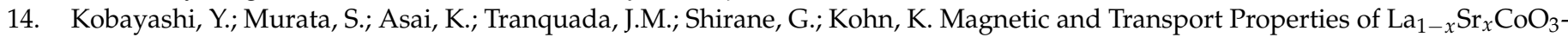
Comparison with $\mathrm{La}_{1-x} \mathrm{Sr}_{x} \mathrm{CoO}_{3}$. J. Phys. Soc. Jpn. 1999, 68, 1011. [CrossRef]

15. Muta, K.; Kobayashi, Y.; Asai, K. Magnetic, Electronic Transport, and Calorimetric Investigations of $\mathrm{La}_{1-x} \mathrm{Sr}_{x} \mathrm{CoO}_{3}$ in $\mathrm{Comparison}$ with $\mathrm{La}_{1-x} \mathrm{Sr}_{x} \mathrm{CoO}_{3}$. J. Phys. Soc. Jpn. 2002, 71, 2784. [CrossRef]

16. Cooper, M.J.; Mijnarends, P.E.; Shiotani, N.; Sakai, N.; Bansil, A. X-ray Compton Scattering; Oxford University Press: Oxford, UK, 2004.

17. Koizumi, A.; Miyaki, S.; Kakutani, Y.; Koizumi, H.; Hiraoka, N.; Makoshi, K.; Sakai, N.; Hirota, K.; Murakami, Y. Study of the e(g) orbitals in the bilayer manganite $\mathrm{La}_{2-2 x} \mathrm{Sr}_{1+2 x} \mathrm{Mn}_{2} \mathrm{O}_{7}$ by using magnetic Compton-profile measurement. Phys. Rev. Lett. 2001, 86, 5589. [CrossRef] [PubMed]

18. Koizumi, A.; Nagao, T.; Sakai, N.; Hirota, K.; Murakami, Y. Coexistence of polaronic and band states in a bilayer manganite from two-dimensional reconstruction of magnetic Compton profiles. Phys. Rev. B 2006, 74, 012408. [CrossRef]

19. Reck, R.A.; Fry, D.L. Orbital and Spin Magnetization in Fe-Co, Fe-Ni, and Ni-Co. Phys. Rev. 1969, 184, 492. [CrossRef]

20. Kobayashi, Y.; Sakurai, Y.; Itou, M.; Sato, K.; Asai, K. Direct Evidence of the Symmetry Change of Co-3d Orbitals Associated with the Spin-State Transition in $\mathrm{LaCoO}_{3}$ by X-ray Compton Scattering. J. Phys. Soc. Jpn. 2015, 84, 114706. [CrossRef]

21. Kobayashi, Y.; Sakurai, Y.; Tsuji, N.; Sato, K.; Asai, K. Symmetry change of Co 3d orbital associated with the 500-K spin crossover accompanied by insulator-to-metal transition in $\mathrm{LaCoO}_{3}$. Phys. Rev. B 2018, 98, 115154. [CrossRef]

22. Okamoto, J.; Miyauchi, H.; Sekine, T.; Shidara, T.; Koide, T.; Amemiya, K.; Fujimori, A.; Saitoh, T.; Tanaka, A.; Takeda, Y.; et al. Direct Evidence of the Symmetry Change of Co-3d Orbitals Associated with the Spin-State Transition in $\mathrm{LaCoO}_{3}$ by $\mathrm{X}_{\text {-ray }}$ Compton Scattering. Magnetic circular x-ray dichroism study. Phys. Rev. B 2000, 62, 4455. [CrossRef] 